Check for updates

Cite this: RSC Adv., 2017, 7, 55408

Received 15th August 2017

Accepted 15th November 2017

DOI: $10.1039 / c 7 r a 09032 c$

rsc.li/rsc-advances

\title{
Tantalum nitride coatings prepared by magnetron sputtering to improve the bioactivity and osteogenic activity for titanium alloy implants
}

\author{
Ruiyan Li, (D) a Yanguo Qin, (DD *a Guancong Liu, (iD ${ }^{a}$ Congxiao Zhang, ${ }^{c}$ Haojun Liang, ${ }^{a}$ \\ Yun'an Qing, ${ }^{a}$ Yanbo Zhang ${ }^{a}$ and Kan Zhang ${ }^{* b}$
}

\begin{abstract}
A good orthopedic load-bearing implant should have both excellent mechanical properties and biological activity. In order to improve the biological activity of Ti6Al4V-based implants, tantalum nitride (TaN) films were deposited on the surface of Ti6Al4V alloy and Si(100) substrates by magnetron sputtering. Characterized by XRD and XPS, it was proved that the uniform cubic $\mathrm{NaCl}$-structure TaN films were successfully prepared on the Ti6Al4V alloy substrate. The TaN film showed higher hardness (19.7 GPa) and elastic modulus (254.4 GPa) compared with HA coatings and Ti6Al4V alloys. We evaluated cytocompatibility and osteogenesis activity of the TaN coatings using rabbit bone marrow stromal cells (BMSCs) cultured on the coated and uncoated samples. The biocompatibility of the films and their effect on cell proliferation were examined by live/dead cell staining and CCK- 8 assay. SEM and cytoskeleton fluorescent staining were used to observe the cell adhesion and morphology on different samples. The effect of the film on osteoblastic activity was assessed by calcium nodule staining and alkaline phosphatase quantitative assays. We demonstrate that TaN film can enhance the adhesion, proliferation, and differentiation of BMSCs. These results suggest that TaN film prepared by magnetron sputtering has great potential to be applied in orthopedics.
\end{abstract}

\section{Introduction}

Ti6Al4V alloy as an implant material is mostly used in orthopedic and dental fields. ${ }^{\mathbf{1}, 2}$ The wide application can be attributed to its excellent biocompatibility, high corrosion resistance, and good mechanical properties. ${ }^{3}$ However, it is known that the surface of Ti6Al4V alloy is generally bioinert, which in turn leads to the instability and aseptic loosening of the implant and results in failure of the implantation. ${ }^{4}$ For the bioinert nature, surface treatment can modify surface topography and chemistry of the Ti6Al4V-based implants and retain or enhance their good mechanical properties. ${ }^{5}$ Besides, deposition of a functional coating onto the surface is also an important and effective method of surface treatment, which can enhance the biocompatibility, osseointegration and mechanical properties of the substrates.

Recently, transition metals like $\mathrm{Ti}, \mathrm{Ta}, \mathrm{Nb}, \mathrm{Hf}$, and $\mathrm{Zr}$ have been reported as metals possessing good biocompatibility and

${ }^{a}$ Department of Joint Surgery of Orthopedic Center, The Second Hospital of Jilin University, Changchun, 130041, China. E-mail: qinyanguo@hotmail.com; Tel: +86-139-04315668

${ }^{b}$ Department of Materials Science, Key Laboratory of Mobile Materials, MOE, State Key Laboratory of Super Hard Materials, Jilin University, Changchun 130012, China. E-mail: zhangkan@jlu.edu.cn; Tel: +86-431-85168444

${ }^{c}$ Department of Stomatology, The First Hospital of Jilin University, Changchun, 130021, China osteoinductivity. ${ }^{6-8}$ They are easily deposited as films used for implant biomaterials. In particular, tantalum (Ta) has gained most interest for a variety of orthopedic implant applications due to its suitability for cell adhesion, proliferation and differentiation..$^{9-11}$ In addition, many studies have shown that Ta is considered as a coating material given its excellent chemical stability, biocompatibility, and osteoconduction. ${ }^{\mathbf{1 2 , 1 3}}$ Currently, most studies focus on regulating the morphology of tantalum, porous tantalum biomaterials were most concerned. However, the biomaterial films should preferably have a high hardness value, resistance to corrosion and mechanical wear, and good adhesion to the substrate which they are deposited on, as well as biocompatibility. Ta thin films would not be the best material for real implant applications since Ta is considered as a soft metal. Ta-based composite coatings can be a good solution to this problem, but there are few reports in the biomedical field. The thin films based on Ta, such as Ta oxides, carbides or nitrides have a high potentiality, since they are hard, and anti-wear and corrosion resistant. Especially tantalum nitride with its unique properties has been applied in many areas, such as diffusion barrier layers in integrated circuits, hard wear-resistant coatings for cutting tools, stable thin film resistors, and nanorod photoanode. ${ }^{\text {14-22 }}$ There are only 3 reports on the biological activity of TaN. Leng et al. reported that TaN film show better blood compatibility than LTI carbon; In 2015, Zhang et al. reported that TaN film was benefit to increase the hardness and modulus of 
the neat $\mathrm{Ti}$, moreover, the resistance of microbiologically which induced corrosion of Ti material was enhanced by the coatings, and their study demonstrated that TaN can be used as coating material for dental applications. ${ }^{23}$ In 2015 , Xu et al. first reported the osteoconductive properties of tantalum nitrides, their study proved that TaN nano ceramic coatings promote apatite formation on the Ti6Al4V alloy. ${ }^{24}$ However, their work just measured the apatite-inducing ability in vitro using a simulated body fluid (SBF). Although SBF has the same inorganic ions as human plasma, it could not reflect the interactions between the coatings and the cells. ${ }^{25}$ There were no studies reported on the compatibility and osteogenic activity of TaN. Moreover, the interactions between cells and TaN film, and the effect of the coating on osteogenesis has not been evaluated in previous work yet. The cell culture experiments are important for evaluating the performance of a biomaterial. ${ }^{26}$ Therefore, we designed cell experiments to evaluate the interactions between cells and TaN film, and the osteogenic activity of the films.

Magnetron sputtering constitutes one of the physical vapor deposition techniques that has been widely used in applications involving the metal-mechanic industry with great successes. For the biomedical field, however, this is an innovative subject with great possibilities which is now under extensive research. ${ }^{27-29}$ In our study, TaN film were fabricated on Ti6Al4V substrate by magnetron sputtering. The mechanical properties were characterized by employing the X-ray diffraction (XRD), X-ray Photoelectron Spectroscopy (XPS) and nanoindenter. The biocompatibility of the film and their effect on cell were examined by live/dead cell staining, CCK- 8 assay, SEM and cytoskeleton fluorescent staining. Meanwhile, the effect of the film on osteoblastic activity was also assessed by calcium nodule staining and alkaline phosphatase quantitative assays.

\section{Experimental}

\section{Deposition of the films}

TaN films were deposited in a DC magnetron sputtering system using a $60 \mathrm{~mm}$-diameter tantalum 99.99\%-pure target. The disk-shaped with a size of $\Phi 10 \times 1.5 \mathrm{~mm}$ medical grade Ti6Al4V alloy and the $25 \times 25 \times 0.5 \mathrm{~mm}^{3} \mathrm{Si}(100)$ wafers were used as the substrate. Before sputter deposition, the Ti6Al4V alloy substrates were ground and then polished consecutively with silicon carbide papers down to 1200 grits. All of the substrates were ultrasonically cleaned in acetone, alcohol and deionized water, and then mounted on the substrate holder. The base pressure in the chamber was evacuated to $4 \times 10^{-4} \mathrm{~Pa}$. Prior to deposition, the target was pre-sputtered with $\mathrm{Ar}^{+}$for 10 minutes to remove the surface oxide layer. A thin $150 \mathrm{~nm}$-thick Ta layer was deposited onto the substrate prior to deposition of the TaN film, in order to improve the film-substrate adhesion. Deposition parameters for the coatings were optimized as follows: a target current of $0.3 \mathrm{~A}$, a substrate bias voltage of $-80 \mathrm{~V}$, a substrate temperature of room temperature, and the nitrogen and Ar flow rate were kept constant at 7 and $60 \mathrm{sccm}$, respectively. The total pressure was kept at $0.8 \mathrm{~Pa}$ during depositions by controlling the pumping speed with a throttling valve, the deposition time was $1 \mathrm{~h}$ and the thickness for films is about $1.5 \mu \mathrm{m}$.

\section{Chemical and structural characterizations}

After deposition, the samples were cut and the center piece was used for characterization. The crystal structure was determined by XRD using a Bragg-Brentano (Bruker D8) diffractometer in a $\theta-2 \theta$ configuration with a $\mathrm{Cu} \mathrm{K} \alpha$ line at $0.15418 \mathrm{~nm}$ as a source. The chemical composition of deposited films was determined by XPS (ESCALAB MKII) with monochromatic Al Ka radiation. In order to remove the surface oxides and contamination from adsorbed hydrocarbons, the samples were sputter cleaned using $800 \mathrm{eV} \mathrm{Ar}^{+}$ions for $20 \mathrm{~min}$ prior to XPS analysis. The nano-scale surface topography and roughness of the TaN film and TC4 sample were measured using an atomic force microscope (AFM).

\section{Mechanical characterization}

The hardness $(H)$ and Young's modulus $(E)$ of the films were evaluated in continuous stiffness measurement mode using a MTS Nanoindenter XP. The films were indented by a Berkovitch type pyramidal diamond tip to a maximum depth of $1000 \mathrm{~nm}$, and at least nine indentations at different places on the film surface were made. Moreover, the curvatures of the samples before and after deposition were also measured by the surface profiler, taking a minimum of eight measurements along two orthogonal surface directions and rejected outlier data points, and the total residual stress was obtained using the Stoney equation.

\section{Cell culture}

The 28-day fetal Japanese White Rabbit (College of veterinary medicine, Jilin University, China) was used to obtained the rabbit bone marrow stromal cells (BMSCs). All the long bones of limbs were dissected from the attached muscles and tissues, and BMSCs were harvested by flushing the bone marrow cavities according to the previous description. ${ }^{30}$ The BMSCs were cultured in Dulbecco's modified Eagle's medium (DMEM; Gibco, USA) supplemental with 10\% fetal bovine serum (FBS; Gibco, USA), $1 \%$ penicillin/streptomycin at $37{ }^{\circ} \mathrm{C}, 5 \% \mathrm{CO}_{2}$. The medium was changed every 2 days. Cells between the third and the fifth passage were used in the follow experiments. The animal procedures were performed in strict accordance with the laboratory animal regulations of the Ministry of Science and Technology of the People's Republic of China (No. [2006] 398) and approved by the ethics committee of the Second Hospital of Jilin University.

\section{Cell attachment and morphology}

The BMSCs were seeded on Ti6Al4V (TC4) and TaN groups at a density of $4 \times 10^{4}$ cells per $\mathrm{ml}$ in 24 -well plates. After $1 \mathrm{~h}$ and $2 \mathrm{~h}$ of incubation, the quantity of adherent cells were determined by CCK-8 kit (CCK-8, Dojindo, Japan). Then, the samples were rinsed with phosphate buffered solution (PBS), fixed with 
4\% paraformaldehyde for $15 \mathrm{~min}$ and stained with 4,6diamidino-2-phenylindole (DAPI, Sigma, USA) for $5 \mathrm{~min}$.

The cytoskeleton organization of the BMSCs were investigated by fluorescent staining. The BMSCs were seeded over TC4 and TaN groups as before. After $1 \mathrm{~h}$ and $2 \mathrm{~h}$ of incubation, the samples were transferred to another new plate and washed by PBS. The remaining cells were fixed in $4 \%$ paraformaldehyde for 15 min at $4{ }^{\circ} \mathrm{C}$. Cells were permeabilized with $0.1 \%$ Triton $\mathrm{X}-100$ for $30 \mathrm{~min}$, while the samples were washed again by PBS. Cytoskeleton was stained with a $50 \mathrm{mg} \mathrm{mL}^{-1}$ fluorescent phalloidin conjugate solution in PBS for 40 minutes at room temperature. Cells were washed several times by PBS to remove the unbound phalloidin conjugate, then the cellular nuclei were stained with DAPI for 5 minutes. The specimens were observed using a confocal laser scanning microscope (OLYMPUS FV1000, USA).

After incubation for $48 \mathrm{~h}$, another group of cell-seeded samples were washed with PBS to discard the non adhesion cells and fixed in $2.5 \% \mathrm{v} / \mathrm{v}$ glutaraldehyde at $4{ }^{\circ} \mathrm{C}$ overnight. Subsequently, the samples were dehydrated through an ethanol series $(30 \%, 50 \%, 75 \%, 80 \%, 90 \%, 95 \%$ and $100 \%)$, criticalpoint dried, and sputtered with Pt. The samples were observed using the FE-SEM (XL-30 ESEM FEG Scanning Electron Microscope FEI Company).

\section{Cell proliferation and viability}

The BMSCs were seeded on different groups at a density of $4 \times$ $10^{4}$ cells per $\mathrm{ml}$ in 24 -well plates. After culturing for $24 \mathrm{~h}$, all cellseeded samples were transferred to new 24-well culture plates and used for analysis cell viability by LIVE/DEAD Cell Imaging Kit staining. LIVE/DEAD Cell Viability Assays (Invitrogen, Life Technologies, Carlsbad, CA, USA) were performed as described in the manual. Samples were imaged using a confocal laser scanning microscope (OLYMPUS FV1000, USA).

The proliferation of BMSCs on each group was determined in 24-well culture plates. Cells were seeded on the samples at $2 \times$ $10^{4}$ cells per ml. At 1, 4 and 7 days, the samples were transferred to new 24-well culture plates respectively, then were washed by PBS for twice. The mixed solution of CCK-8 and DMEM $(10: 100)$ was added to wells and incubated at $37^{\circ} \mathrm{C}$ for $2 \mathrm{~h}$. Cell proliferation was measured by testing optical density (OD) values at $450 \mathrm{~nm}$ using a microplate reader (Varioskan Flash, Thermo Scientific). The cell number was correlated with the OD values.

To evaluate the apoptosis, the BMSCs were seeded on TC4 and TaN groups at a density of $2 \times 10^{4}$ cells per $\mathrm{ml}$ in 24 -well plates, and cell culture plate group was set as control. After incubation for 3 and 7 days, the cell-seeded samples were washed with PBS and lysed using RIPA Lysis Buffer (Beyotime, China). The protein concentration was determined using the BCA assay kit (Beyotime, China), and equivalent amounts of protein were separated and analyzed with a SDS-PAGE assay, probing with primary antibodies. Antibodies against caspase-3 (ab208161, abcam, USA; $1: 500$ dilution) and $\beta$-actin (ab6276, abcam, USA; 1:2000 dilution). The membranes were then incubated with the respective horseradish peroxidaseconjugated goat anti-mouse IgG secondary antibodies (Daixuan Bio., China) at room temperature for $2 \mathrm{~h}$. Immunoreactivity bands were detected using an FR-1800 Luminescent and fluorescent biological image analysis system (Furi Science \& Technology Co., China).

\section{Alkaline phosphates (ALP) activity assay and alizarin red staining}

The BMSCs were seeded on different groups at a density of $1 \times$ $10^{5}$ cells per $\mathrm{ml}$ in 24-well culture plates. After cell adherence, the cells were cultured using the osteogenic medium (complete medium supplemented with $50 \mathrm{mg} \mathrm{L}{ }^{-1}$ ascorbic acid, $108 \mathrm{M}$ dexamethasone and $10 \mathrm{mM}$ b-glycerol phosphate). The medium was changed every 2 days.

After osteogenic medium treatment for 7 days. ALP staining was carried out using a BCIP/NBT Alkaline Phosphatase Color Development Kit (Beyotime, China). To quantify the alkaline phosphatase (ALP) activity, the samples were washed thrice with PBS and disrupted by RIPA Lysis Buffer (Beyotime, China). ALP activity was detected using a commercially available kit (Beyotime, China), according to the manufacturer's instructions. Units per microgram of protein content ALP activity was measured by testing optical density (OD) values at $405 \mathrm{~nm}$. Total protein content was calculated using the BCA assay kit (Beyotime, China). ALP activity was expressed as the OD values at $405 \mathrm{~nm}$ per milligram of total proteins.

After incubation of 7 and 14 days, the BMSCs of each group were fixed and stained with alizarins $(\mathrm{pH}=4.2)$ at room temperature in the dark, observation and images were captured with a zoom stereo microscope.

\section{Statistical analysis}

The results were presented as the means $\pm \mathrm{SD}$ for each group. $T$ tests followed by Sidak-Bonferroni method were used to perform the statistical analysis among different groups. $P$ value $<0.05$ was considered statistically significant.

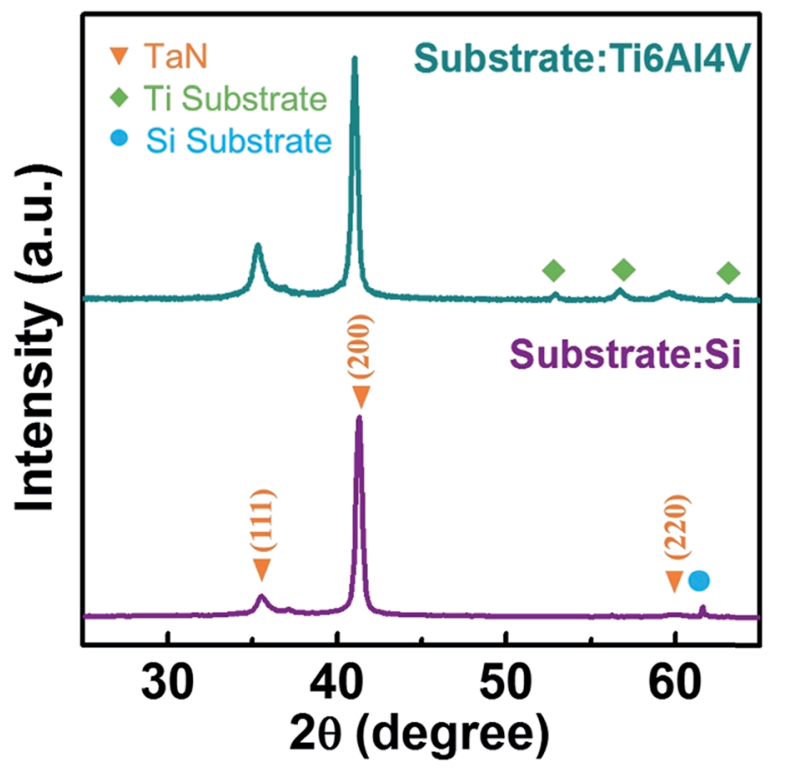

Fig. 1 XRD patterns of the TaN films deposited on the Si and Ti6Al4V. 


\section{Results and discussion}

Fig. 1 showed the X-ray diffraction $\theta-2 \theta$ spectra over the measured $2 \theta=25-65^{\circ}$ range for the TaN film deposited onto
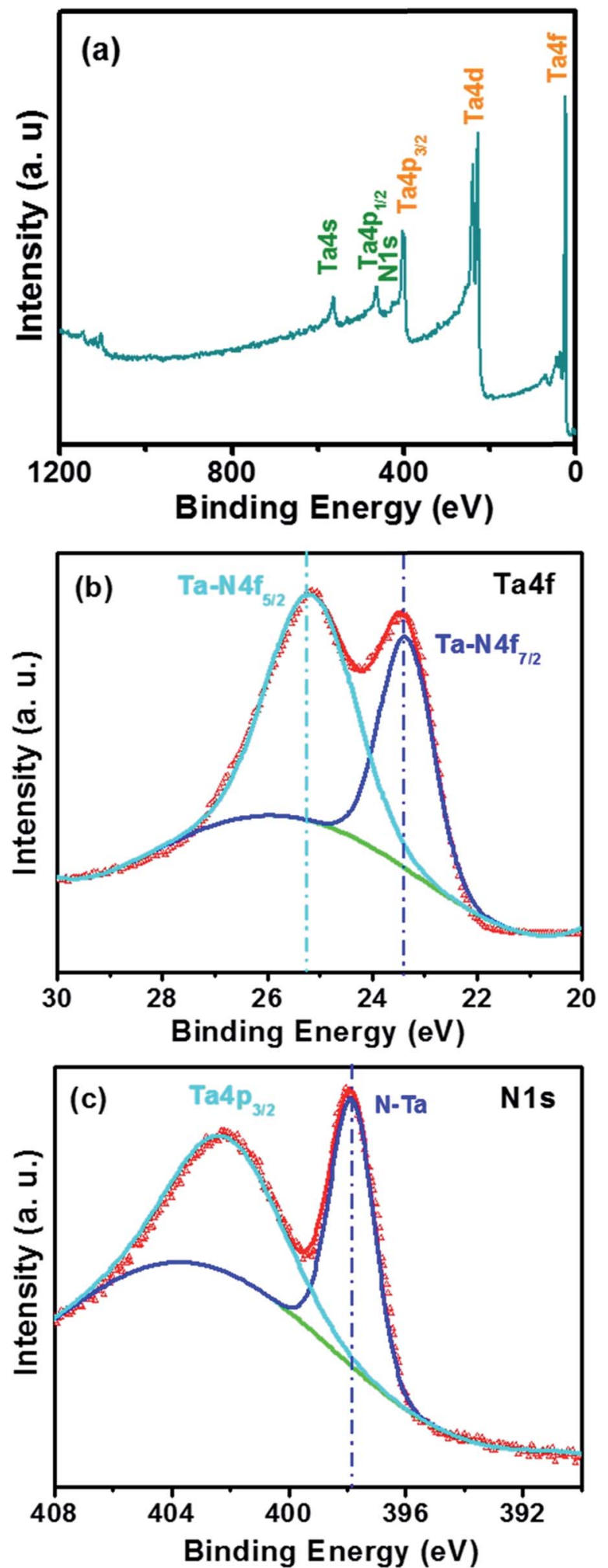

Fig. 2 The XPS survey spectra (a), XPS spectra for Ta $4 f$ and (b) N 1s (c) for TaN films deposited on the Si.
Si(100) and Ti6Al4V alloy substrates. All the films showed diffraction peaks at $35.5^{\circ}, 41.3^{\circ}$, and $59.9^{\circ}$ which were assigned to (111), (200), and (220) reflections of the cubic NaCl-structure TaN (JCPDF 49-1283), respectively. The peaks were at $0.3-0.4^{\circ}$ lower $2 \theta$ than the literature values indicated by vertical dashdotted lines in Fig. 1, which could be ascribed to the compressive stress in the obtained film, caused by bombarding ion energy.

The XPS survey spectra for TaN film was shown in Fig. 2(a), in which the spectral signature of tantalum and nitrogen were observed. Fig. 2(b) and (c) exhibited the detailed scan XPS corelevel spectra in the energy region of Ta $4 \mathrm{f}$ and $\mathrm{N} 1 \mathrm{~s}$ for TaN films.

In Fig. 2(b), the curve exhibited two distinct peaks for Ta $4 \mathrm{~d}_{5 / 2}$ and Ta $4 \mathrm{f}_{7 / 2}$ spectra: one locates at $25.3 \mathrm{eV}$ and another at $23.4 \mathrm{eV}$, and the peak separation maintained a constant of $1.9 \mathrm{eV}$, which was in good agreement with the values reported in the literature. ${ }^{31,32}$ For the Ta $4 \mathrm{~d}_{5 / 2}$ peak, which was considered to be associated with the Ta-N bonding, since the Ta $4 \mathrm{f}$ peaks relative to that (centered at $22.1 \mathrm{eV}$ (ref. 33 and 34)) of the pure Ta film deposited in pure Ar discharging is shifted to higher binding energies, reflecting a charge transfer from tantalum to nitrogen in the nitrogenization process. While for the Ta $4 \mathrm{f}_{7 / 2}$ peak with the binding energy of $23.4 \mathrm{eV}$, which was very close with the previous report, in which, the researchers have reported a value of $\sim 23.5 \mathrm{eV}$ for the Ta $4 \mathrm{f}_{7 / 2}$ band for a cubic TaN IBAD film. ${ }^{35}$ This also agreed well with our XRD result. The
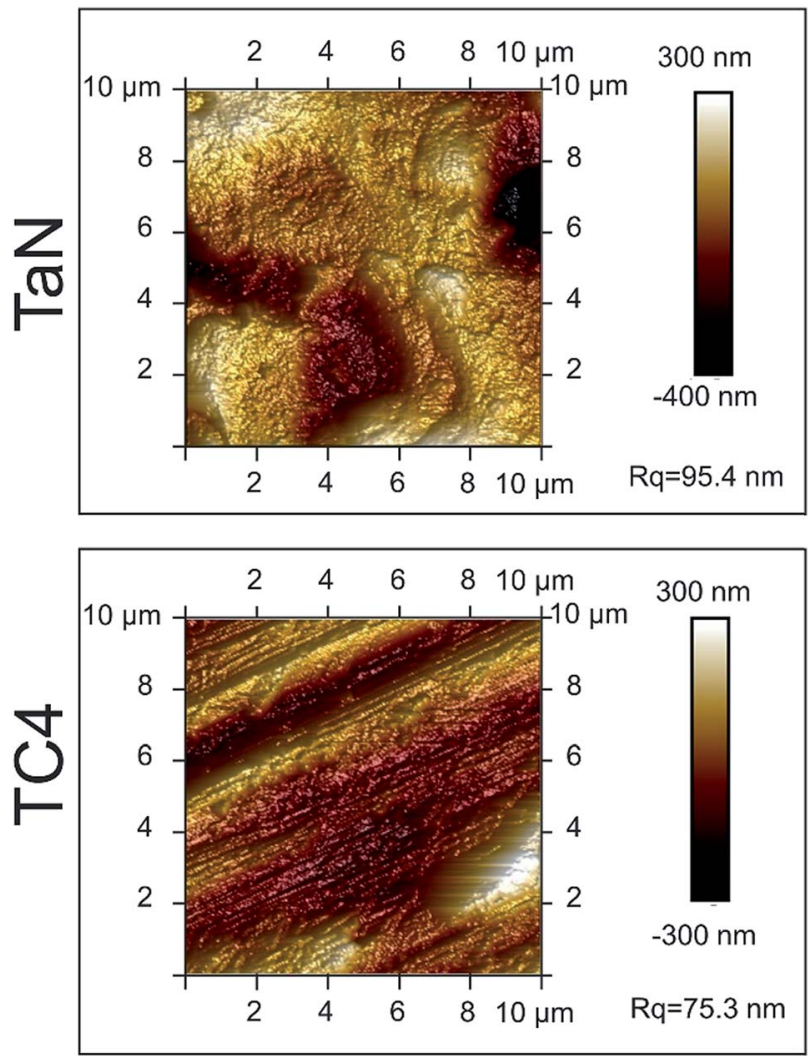

Fig. 3 Representative AFM images for the surfaces of TaN film and TC4. 
Table 1 The deposition rate, overall film composition, hardness $H$ and elastic modulus $E$ for the film deposited on the Si

\begin{tabular}{|c|c|c|c|c|c|c|}
\hline Sample & Ta (at\%) & $\mathrm{N}$ (at\%) & $\mathrm{O}(\mathrm{at} \%)$ & $H(\mathrm{GPa})$ & $E(\mathrm{GPa})$ & $\begin{array}{l}\text { Deposition rate } \\
\left(\mathrm{nm} \mathrm{min}{ }^{-1}\right)\end{array}$ \\
\hline $\mathrm{TaN}$ & 58.5 & 40.2 & 1.3 & 19.7 & 254.4 & 28.6 \\
\hline
\end{tabular}
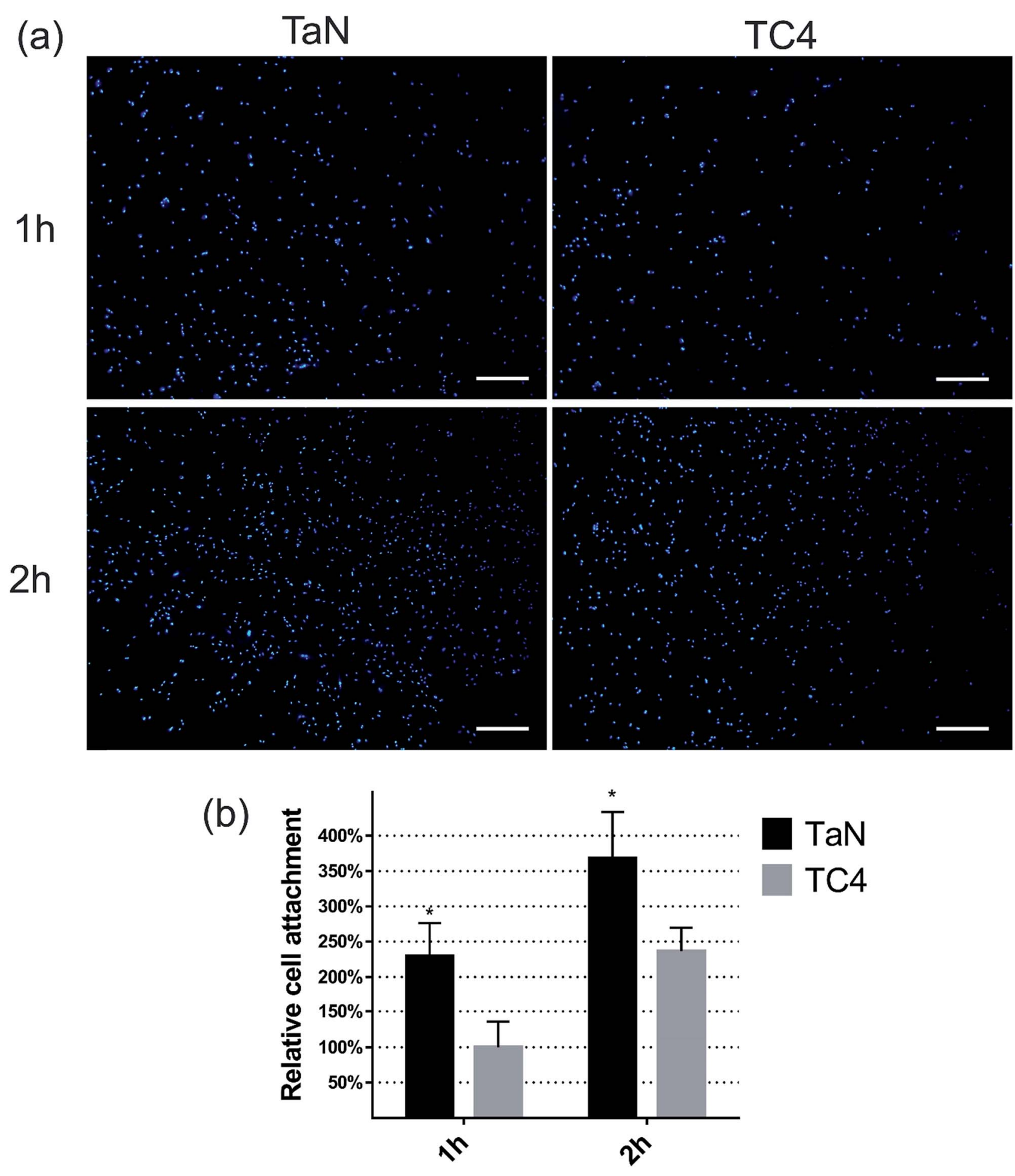

Fig. 4 The fluorescence images of nuclei (a) and the relative cell attachment (b) expressed as a percentage of different groups after 1 and $2 \mathrm{~h}$ culture. Scale: $500 \mu \mathrm{m}$ (* indicates statistical significance $p<0.05$ vs. TC4). 
existence of the Ta-N bonding in the film was further confirmed by the $\mathrm{N}$ 1s spectra in Fig. 2(c), the formation of Ta nitride was corroborated by the emergence of a new $\mathrm{N}$ (1s) feature at $397.8 \mathrm{eV}$. The Ta $\left(4 \mathrm{p}_{3 / 2}\right)$ feature overlapped this portion of the $\mathrm{N}$ (1s) spectrum, with a broad $\mathrm{Ta}\left(4 \mathrm{p}_{3 / 2}\right)$ peak at binding energy of 400.9-404 eV. ${ }^{36-38}$

Fig. 3 showed the representative AFM images of the investigated samples. For the TC4 group, many nicks emerged on the surface of the sample, which was caused by polishing. Whereas for the TaN group, a thick TaN film was deposited on the substrate, and the nicks were covered by the film. In addition, the AFM characterization demonstrates that the deposition of TaN increase the roughness of sample. Furthermore, the surface roughness value $\left(R_{\mathrm{q}}\right)$ of TaN film is $95.4 \mathrm{~nm}$, which is higher than the value of TC4 $(74.3 \mathrm{~nm})$.

To understand properties of the film better, the hardness and elastic modulus calculated based on load-displacement nanoindentation curves using the method proposed by Oliver and Pharr are listed in Table $1 .^{39}$ The hardness values for the TaN film was around 19.7 GPa, significantly higher than the nanohardness of reported values for hydroxyapatite (HA) coatings, ${ }^{40}$ range in 1.5 and $5 \mathrm{GPa}$, and Ti-6Al-4V alloys, around 4-
$5 \mathrm{GPa}^{41}$ In addition, the TaN film had the highest elastic modulus (254.4 GPa), also much higher than those reported values of HA coatings ${ }^{40}$ and Ti6Al4V alloys. ${ }^{41}$

The cell adhesion is a key feature of the biocompatibility, which can be summarized from the amount and morphology of adherent cells. The amount of cells which adhered on different surface was detected by nucleus fluorescent staining and CCK-8 kit, and shown in Fig. 4(a) and (b). From Fig. 4(a), lots nuclei which were stained as blue dots can be observed on different surfaces, and much more blue dots merged on TaN group compared with TC4 group at both 1 and $2 \mathrm{~h}$. The relative cell attachment was shown in the Fig. 4(b). At $1 \mathrm{~h}$, the number of cells on TaN group was significant much more than the TC4 group ( $>2$-fold). At $2 \mathrm{~h}$, the amount of adherent cells increased with incubation time of all groups, but the number of cells on TaN group was still about 1.5-fold the number of TC4 group.

To further investigate cell adhesion behavior, the initial cell morphology was observed by F-actin (green), and nucleus (blue) fluorescence staining, and shown in Fig. 5. From the images, it can be seen that BMSCs adhered on both TaN and TC4 groups, and the cell morphology became better with incubation time. After $1 \mathrm{~h}$ incubation, the cells displayed well expanding on TaN

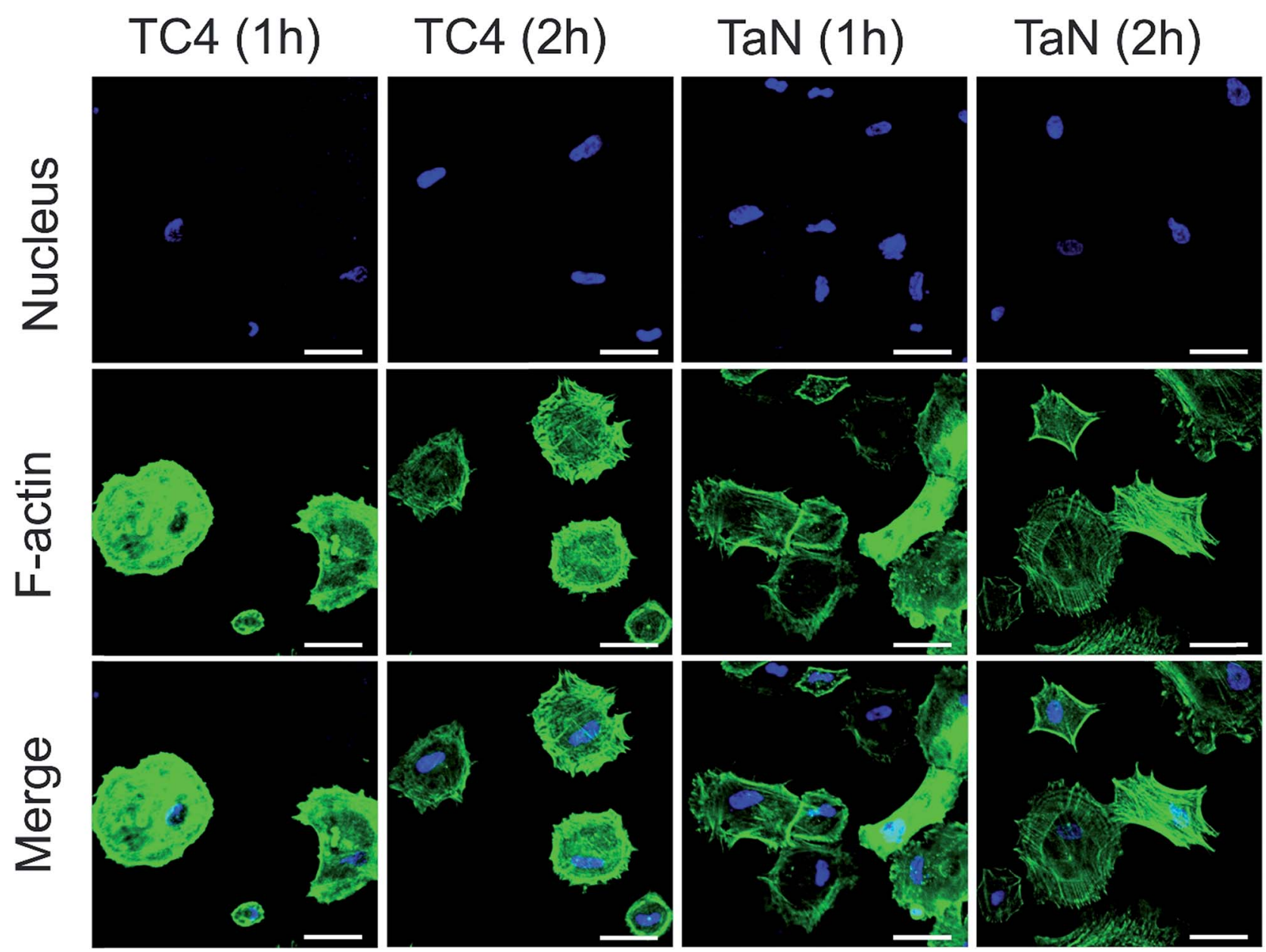

Fig. 5 Confocal laser scanning microscopy images for cytoskeletal morphology of BMSCs on different groups (green, phalloidin for F-actin; blue, DAPI for nucleus). Scale: $100 \mu \mathrm{m}$. 
group with typical spindle-shaped morphology of BMSCs, by contrast, the cells appeared on TC4 group showed shrinking circular shape. After $2 \mathrm{~h}$ incubation, cells on TaN group exhibited obvious rearranged cytoskeleton in the cytoplasm, and the microfilaments of the cytoskeleton were clear and regular. While the cells on the TC4 group still displayed shrinking circularshape morphology, moreover, the microfilaments of cells on TC4 group were blurred and disorderly. It can be concluded that the cell status on TaN group was better than that on TaN group, which supported that TaN film promoted early cell adhesion. Initial adhesion affects subsequent proliferation and differentiation of stem cells. ${ }^{42}$ The result of relative cell attachment and the fluorescent staining images of BMSCs revealed that TaN film can induce migration of BMSCs to the surface of implant, which would give rise to better osseointegration.

Fluorescence staining can only display the cytoskeletons and nuclei, while the surface morphology of TaN film could not observed under laser scanning confocal microscopy. The SEM images can clearly show cell morphology and material surface topography at the same time, which are helpful for further study of the cell-surface interactions. Fig. 6 showed SEM images of cells after culturing for $48 \mathrm{~h}$ on the surface of each group. The cell contour and topography could be clearly seen from Fig. 6 . Cells were attaching on the surface of TC4 and TaN groups with a good morphology. Moreover, cells on TaN films showed more filopodias (shows as red arrow) at high magnification. Combined with the results of fluorescence staining, it can be concluded that the TaN film provided an outstanding condition for the attachment and spreading of BMSCs. Many studies have reported that surface roughness significantly affected the cell response to the material, and the surface roughness has a positive influence on cell adhesion..$^{43-45}$ Combined the result of AFM images, the TaN film significant increased the roughness of the substrates, which can be the reason for the better cell adhesion on TaN group.

Biocompatibility is a basic requirement to measure whether a material can be used as a biomaterial. BMSCs could proliferate in vitro and could be induced to differentiate into more

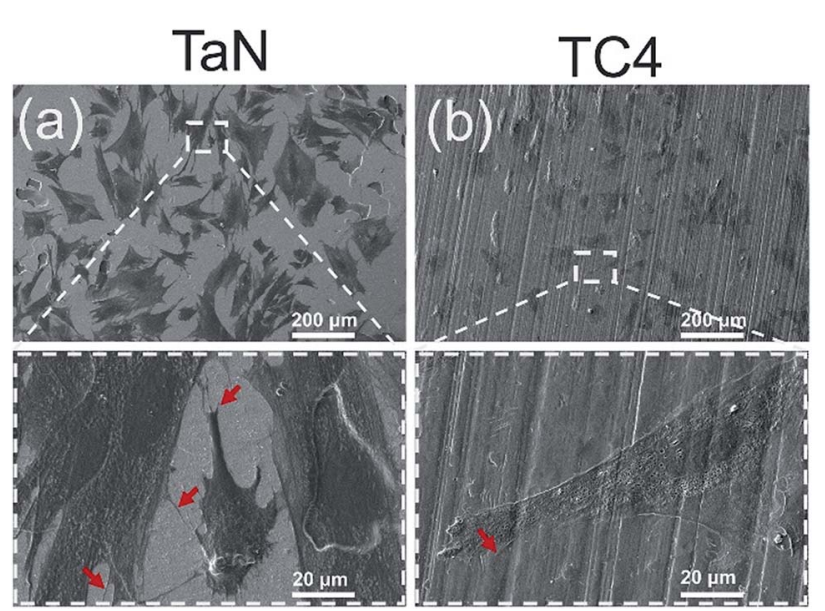

Fig. 6 SEM image of BMSCs cultured on TaN (a) and TC4 (b) samples for 2 days. specialized cells. ${ }^{46}$ The interaction of cells with the surface of the material can reflect the biocompatibility of the material. Thus, the BMSCs were cultured on samples to evaluate the cytocompatibility of coated and uncoated samples. Fig. 7 showed the confocal laser scanning microscope images of the BMSCs cultured on the TC4 and TaN groups for $24 \mathrm{~h}$. The dead cells (red dot, white circle) and live cells (green dot) were stained and shown in the picture. There was almost no red dots on TaN group, while the TC4 group had lots of red dots. Meanwhile, it can be seen that there were much more green dots on TaN group than TC4 group. Based on this result, it is means the TC4 coated with TaN film showed the better cytocompatibility in vitro than TC4.

However, the growth of cells on the coating is a long-term process. Cell proliferation experiments are essential importance to understand the interactions of cells and film, and the cytotoxicity for the film can be observed by the cell proliferation on the coating. In this research, the proliferation of the cells cultured on the TC4 and TaN groups was evaluated by CCK-8 kit, and the result is displayed in Fig. 8. The OD value increased over the incubation time in all groups. There was almost no
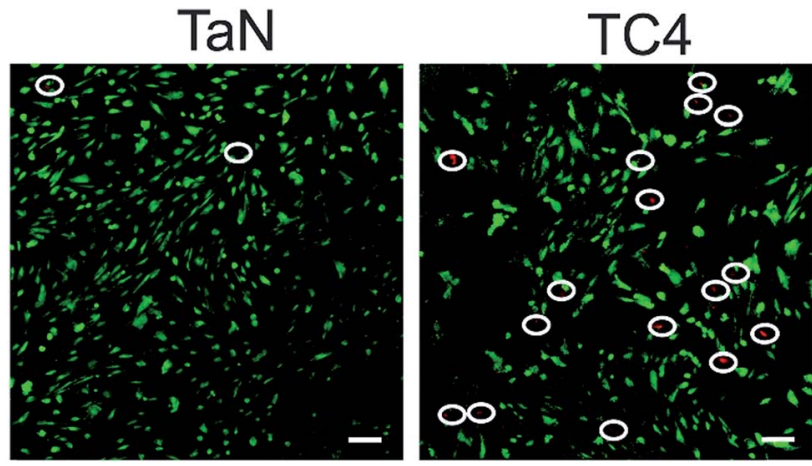

Fig. 7 Confocal microscopy images of BMSCs that were cultured on different groups with live/dead staining (green colour: live cells, red colour: dead cells). Scale: $100 \mu \mathrm{m}$.

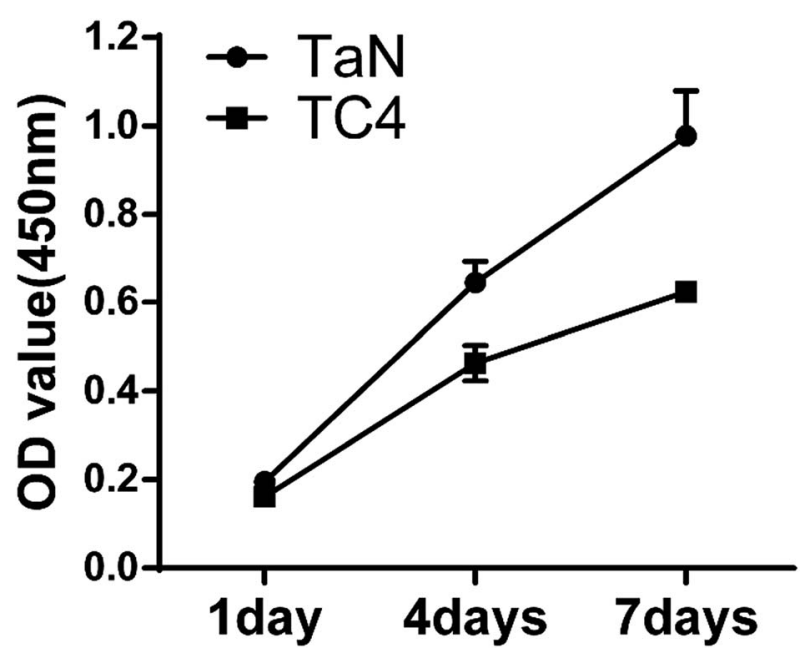

Fig. 8 Cell proliferation of BMSCs on different groups measured by CCK- 8 assay after culture 1,4 and 7 days. 
difference between the two groups at 1 day. However, it could be seen the difference of two groups became larger and larger after 1 day based on the curve. In addition, the proliferation rate of cells cultured on TaN films was higher than that cultured on TC4 groups at both time points ( 4 days and 7 days $)(P<0.05)$. The higher proliferation rate related to TaN group which indicated that TaN films can promote the cell proliferation compared with TC4.

\section{3 days TaN TC4 Control \\ Caspase-3 \\ $\beta$-actin

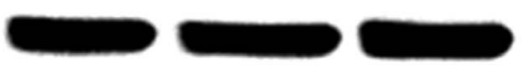

\section{7 days}

Caspase-3

\section{$\beta$-actin}

\section{TaN}

TC4 Control

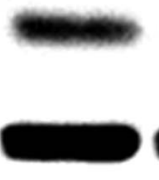

Fig. 9 The expression of caspase-3 for TaN group, TC4 group, and the control group.
Foreign body reaction is a major problem for implantation, which will induce immune response, and then cause apoptosis. The expression of caspase- 3 has been identified as an early indicator of apoptosis, and it acts as an executioner in the final steps of the apoptotic program. ${ }^{\mathbf{4 4 , 4 7 , 4 8}}$ The expression of caspase3 was detected to assess the immune response, and shown in Fig. 9. Obviously, the immunoreactivity bands of all groups is similar, which mean that the expression of caspase- 3 of all groups has no obvious difference, revealed the TaN film did not cause apoptosis. Combined with the live/dead cells staining images and cell proliferation curves, suggest that TaN film has excellent biocompatibility.

The osteogenesis differentiation of the cells reflected the biological activity of the material and may better explain the prospect of the materials as orthopedic implants. ALP is widely used as an early osteoblast marker, and its increased activity is associated with osteoblastic differentiation. ${ }^{49}$ Fig. 10(a) showed the ALP staining of BMSCs cultured on different groups for 7 days, in which the ALP-positive areas was stained as blue-purple spots. The percentage of the ALP-positive areas on TaN group was obvious larger than that on TC4 group, revealed that TaN film induced the expression of ALP. Moreover, the ALP activity of BMSCs on different groups was evaluated and shown in Fig. 10(b). Obviously, the ALP activity of TaN group was significantly higher than TC4 group, which also demonstrated that TaN film enhanced the ALP activity of BMSCs.

In the late stage of osteogenesis, extracellular matrix will mineralize as calcium nodules, so that the calcium deposition is an important direct indicator of osteogenesis, and can be stained as red spots by alizarin red reagents. The Fig. 11 showed
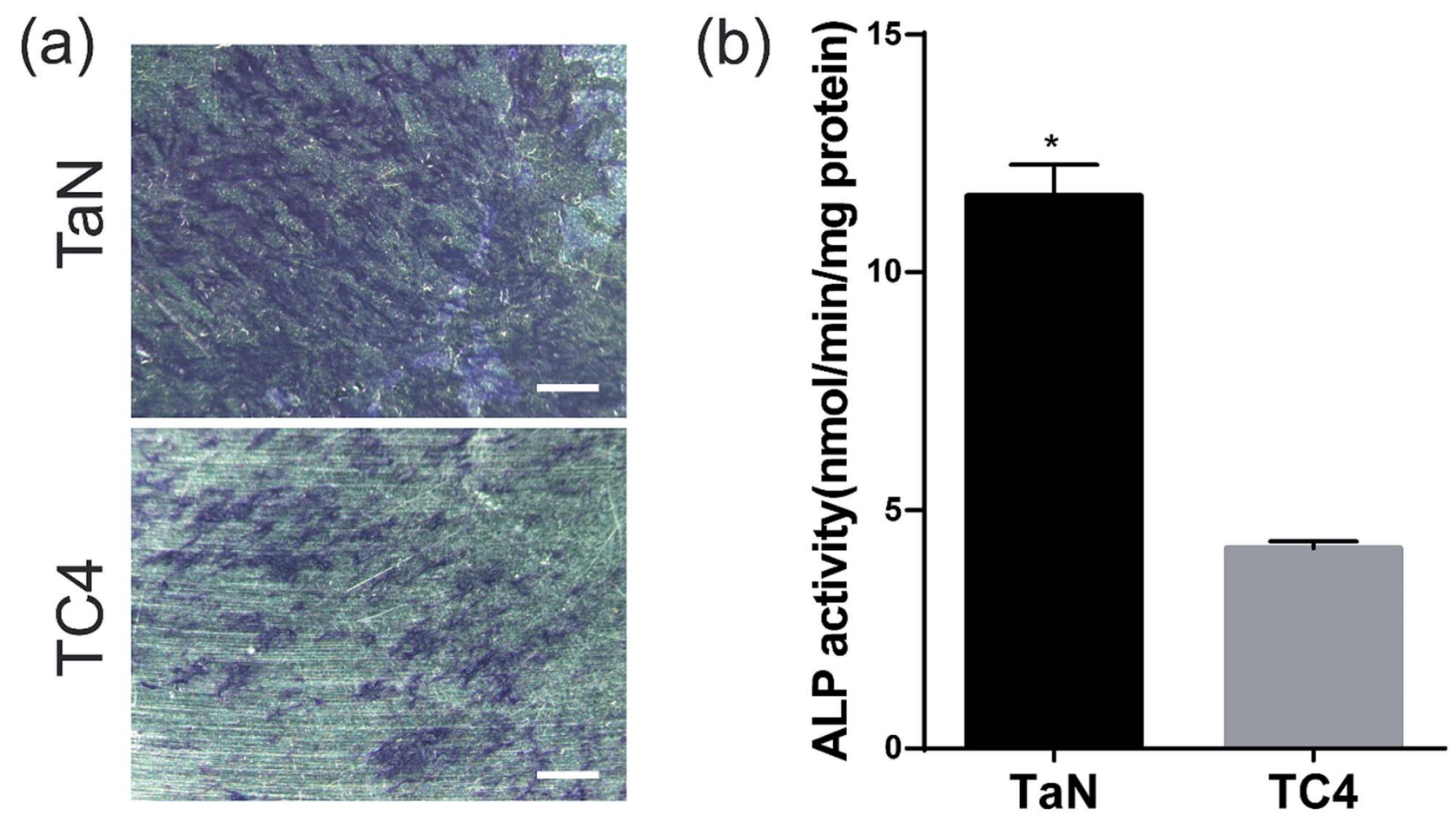

Fig. 10 (a) ALP staining images of different groups at 7 days. Scale: $100 \mu \mathrm{m}$; (b) quantification of ALP activity for all groups at 7 days (* indicates statistical significance $p<0.05$ vs. TC4). 


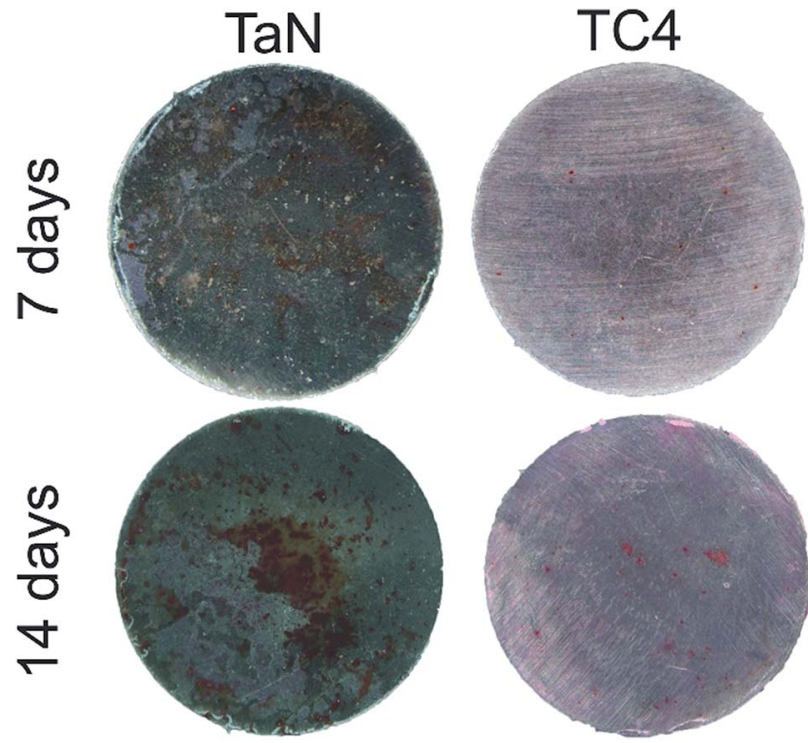

Fig. 11 Alizarin red staining images of different groups at 7 and 14 days.

alizarin red staining of BMSCs on different groups after osteogenic induction for 7,14 days. It can be seen that a few calcium nodules appears in all groups at 7 days, and, its number and distribution increase after induction for 14 days. With the increasing culture time to 14 days, the areas and number of calcium nodules in the TaN group were markedly larger than that in TC4 group. It can be deduced that TaN film substantially promoted mineralized nodules formation of BMSCs. The findings are consistent with the results of ALP staining and quantitation of ALP activity, demonstrated that TaN film can enhance the osteogenic activity in vitro.

\section{Conclusions}

In this study, TaN film was successfully deposited on the surface of Ti6Al4V alloy (TC4) and Si (100) substrates via magnetron sputtering. Biomaterial characterization, biocompatibility assays and osteogenesis assays were used to evaluate the performance of the film. The results reveal that uniform TaN film was deposited on the substrate with NaCl-type structure, and showed higher hardness (19.7 GPa) and elastic modulus (254.4 GPa) compared with Ti6Al4V alloys. Biocompatibility assays demonstrated that TaN film can induce migration of BMSCs to substrate, and provide outstanding condition for cell adhesion and proliferation, particularly, cells on TaN group grow much faster than TC4 group. Furthermore, osteogenesis assays revealed much more calcium nodules emerge on TaN group compared with TC4 group, and the TaN film enhanced the ALP activity of BMSCs. To sum up, TaN film has excellent mechanical properties and biocompatibility, and enhance the bioactivity of osteogenesis. Our study suggests that TaN film may be a promising method to enhance the osseointegration of Ti6Al4V-based implants.

\section{Conflicts of interest}

The authors declare that they have no competing interests.

\section{Acknowledgements}

The support from National Natural Science Foundation of China (Grant No. 51602122), the National Key Research and Development Program of China (No. 2016YFA0200400), China postdoctoral Science Foundation (Grant No. 2016M600229), Norman Bethune Program of Jilin University (Grant No. 2012216), Science and Technology Development program of Jilin province (Grant No. 20150414006GH, 20170520120JH), a special fund project of Jilin provincial industrial innovation (No. 2016C037), is highly appreciated.

\section{Notes and references}

1 C. Elias, J. Lima, R. Valiev and M. Meyers, JOM, 2008, 60, 4649.

2 D. L. Wise, D. J. Trantolo, K.-U. Lewandrowski, J. D. Gresser, M. V. Cattaneo and M. J. Yaszemski, Biomaterials engineering and devices: human applications, Springer, 2000.

3 D. M. Brunette and B. Chehroudi, J. Biomech. Eng., 1999, 121, 49-57.

4 N. G. Durmus and T. J. Webster, Nanomedicine, 2012, 7, 791793.

5 M. Kulkarni, A. Mazare, P. Schmuki, A. Iglic and A. Seifalian, Nanomedicine, 2014, 111, 111.

6 H. Kawahara, S. Ochi, K. Tanetani, K. Kato, M. Isogai, H. Mizuno, H. Yamamoto and A. Yamagami, J. Jpn. Soc. Dent. Appar. Mater., 1963, 4, 65-75.

7 S. Steinemann, Evaluation of biomaterials, 1980.

8 H. Matsuno, A. Yokoyama, F. Watari, M. Uo and T. Kawasaki, Biomaterials, 2001, 22, 1253-1262.

9 B. R. Levine, S. Sporer, R. A. Poggie, C. J. Della Valle and J. J. Jacobs, Biomaterials, 2006, 27, 4671-4681.

10 J. D. Bobyn, K.-K. Toh, S. A. Hacking, M. Tanzer and J. J. Krygier, J. Arthroplasty, 1999, 14, 347-354.

11 D. M. Findlay, K. Welldon, G. J. Atkins, D. W. Howie, A. C. Zannettino and D. Bobyn, Biomaterials, 2004, 25, 2215-2227.

12 F. Meng, Z. Li and X. Liu, Surf. Coat. Technol., 2013, 229, 205209.

13 Z. Tang, Y. Xie, F. Yang, Y. Huang, C. Wang, K. Dai, X. Zheng and X. Zhang, PLoS One, 2013, 8(6), e66263.

14 Y. Zhang, Y. Zheng, Y. Li, L. Wang, Y. Bai, Q. Zhao, X. Xiong, Y. Cheng, Z. Tang, Y. Deng and S. Wei, PLoS One, 2015, 10, e0130774.

15 L. Wang, N. T. Nguyen, X. Zhou, I. Hwang, M. S. Killian and P. Schmuki, ChemSusChem, 2015, 8, 2615-2620.

16 A. Rugge, J. S. Park, R. G. Gordon and S. H. Tolbert, J. Phys. Chem. B, 2005, 109, 3764-3771.

17 F. Mafune, Y. Tawaraya and S. Kudoh, J. Phys. Chem. A, 2016, 120, 4089-4095.

18 Y. Li, L. Zhang, A. Torres-Pardo, J. M. Gonzalez-Calbet, Y. Ma, P. Oleynikov, O. Terasaki, S. Asahina, M. Shima, 
D. Cha, L. Zhao, K. Takanabe, J. Kubota and K. Domen, Nat. Commun., 2013, 4, 2566.

19 P. Kroll, T. Schroter and M. Peters, Angew. Chem., Int. Ed. Engl., 2005, 44, 4249-4254.

20 Y. Fukasawa, K. Takanabe, A. Shimojima, M. Antonietti, K. Domen and T. Okubo, Chem.-Asian J., 2011, 6, 103-109.

21 H. R. Chen, Y. C. Chen, T. C. Chang, K. C. Chang, T. M. Tsai, T. J. Chu, C. C. Shih, N. C. Chuang and K. Y. Wang, Nanoscale Res. Lett., 2014, 9, 177.

22 J. Bryner, D. M. Profunser, J. Vollmann, E. Mueller and J. Dual, Ultrasonics, 2006, 44(suppl. 1), e1269-1275.

23 Y. Zhang, Y. Zheng, Y. Li, L. Wang, Y. Bai, Q. Zhao, X. Xiong, Y. Cheng, Z. Tang and Y. Deng, PLoS One, 2015, 10, e0130774.

24 J. Xu, L. Liu, P. Munroe and Z.-H. Xie, J. Mater. Chem. B, 2015, 3, 4082-4094.

25 T. Kokubo and H. Takadama, Biomaterials, 2006, 27, 29072915.

26 M. Vandrovcová and L. Bacakova, Physiol. Res., 2011, 60, 403. 27 L. Bai, R. Hang, A. Gao, X. Zhang, X. Huang, Y. Wang, B. Tang, L. Zhao and P. K. Chu, Appl. Surf. Sci., 2015, 355, 32-44.

28 Z. E. Sánchez-Hernández, M. A. Dominguez-Crespo, A. M. Torres-Huerta, E. Onofre-Bustamante, J. A. Adame and H. Dorantes-Rosales, Mater. Charact., 2014, 91, 50-57.

29 S. C. Velasco, V. Lopez, C. A. Alves, A. Cavaleiro and S. Carvalho, Corros. Sci., 2014, 80, 229-236.

30 Z. Y. Zhang, S. H. Teoh, M. S. Chong, J. T. Schantz, N. M. Fisk, M. A. Choolani and J. Chan, Stem Cells, 2009, 27, 126-137.

31 J.-C. Chuang and M.-C. Chen, Thin Solid Films, 1998, 322, 213-217.

32 S. Badrinarayanan and S. Sinha, J. Appl. Phys., 1991, 69, 1141-1146.

33 J. Tong, D. Martini, N. Magtoto and J. Kelber, J. Vac. Sci. Technol., B, 2003, 21, 293-300.
34 J. Moulder, W. Stickle, P. Sobol and K. Bomben, Handbook of X-Ray Photoemission Spectroscopy, Perkin-Elmer, 1995.

35 Q. Zhang, X. Mei, D. Yang, F. Chen, T. Ma, Y. Wang and F. Teng, Nucl. Instrum. Methods Phys. Res., Sect. B, 1997, 127, 664-668.

36 Y.-L. Kuo, J.-J. Huang, S.-T. Lin, C. Lee and W.-H. Lee, Mater. Chem. Phys., 2003, 80, 690-695.

37 E. Engbrecht, Y.-M. Sun, S. Smith, K. Pfiefer, J. Bennett, J. White and J. Ekerdt, Thin Solid Films, 2002, 418, 145-150.

38 R. Nyholm, A. Berndtsson and N. Martensson, J. Phys. C: Solid State Phys., 1980, 13, L1091.

39 W. C. Oliver and G. M. Pharr, J. Mater. Res., 1992, 7, 15641583.

40 A. Dey, A. K. Mukhopadhyay, S. Gangadharan, M. K. Sinha and D. Basu, J. Mater. Sci., 2009, 44, 4911-4918.

41 L. Murr, S. Quinones, S. Gaytan, M. Lopez, A. Rodela, E. Martinez, D. Hernandez, E. Martinez, F. Medina and R. Wicker, J. Mech. Behav. Biomed. Mater., 2009, 2, 20-32.

42 L. Bacakova, E. Filova, M. Parizek, T. Ruml and V. Svorcik, Biotechnol. Adv., 2011, 29, 739-767.

43 J.-W. Park, Y.-J. Kim, C. H. Park, D.-H. Lee, Y. G. Ko, J.-H. Jang and C. S. Lee, Acta Biomater., 2009, 5, 3272-3280.

44 A. Wennerberg and T. Albrektsson, Clin. Oral Implants Res., 2009, 20, 172-184.

45 M. M. Stevens and J. H. George, Science, 2005, 310, 11351138.

46 A. Bakhtina, M. Tohfafarosh, A. Lichtler and T. L. Arinzeh, Vitro Anim. Cell Dev. Biol., 2014, 50, 251-260.

47 B.-Z. Cai, F.-Y. Meng, S.-L. Zhu, J. Zhao, J.-Q. Liu, C.-J. Liu, N. Chen, M.-L. Ye, Z.-Y. Li and J. Ai, Toxicol. Lett., 2010, 193, 173-178.

48 G. S. Salvesen, Cell Death Differ., 2002, 9, 3-5.

49 R. Marom, I. Shur, R. Solomon and D. Benayahu, J. Cell. Physiol., 2005, 202, 41-48. 